\title{
Oscillation mode transformation of edge magnetoplasmons in two-dimensional electron system on liquid-helium surface
}

\author{
Shuji Yamanaka ${ }^{1}$, Toshikazu Arai ${ }^{2 *}$, Anju Sawada ${ }^{2}$, Akira Fukuda $^{3}$, and Hideki Yayama ${ }^{1}$ \\ ${ }^{1}$ Department of Physics, Graduate School of Sciences, Kyushu University, Fukuoka 812-8581, Japan \\ ${ }^{2}$ Research Center for Low Temperature and Materials Sciences, Kyoto University, Kyoto 606-8502, Japan \\ E-mail: toshikaz@tohtech.ac.jp \\ ${ }^{3}$ Department of Physics, Hyogo College of Medicine, Nishinomiya, Hyogo 663-8501, Japan
}

Received March 12, 2013

\begin{abstract}
We measured the resonance spectra of edge magnetoplasmon (EMP) oscillations in a two-dimensional (2D) electron system located on a liquid-helium surface below $1.1 \mathrm{~K}$. Systematic measurements of the resonance frequency and the damping rate as a function of the lateral confinement electric field strength shows clear evidence of the oscillation mode transformation. A pronounced change corresponding to the mode transformation was observed in the damping rate. When 2D electrons are confined in a strong lateral electric field, the damping is weak. As the lateral confinement electric field is reduced below a certain threshold value, an abrupt enhancement of the damping rate is observed. We hypothesize that the weak damping mode in the strong lateral confinement electric field is the compressive density oscillation of the electrons near the edge (conventional EMP) and the strong damping mode in the weak confinement field is the coupled mode of conventional EMP and the boundary displacement wave (BDW). The observation of the strong damping in the BDW-EMP coupled mode is a manifestation of the nearly incompressible feature of strongly interacting classical electrons, which agrees with earlier theoretical predictions.
\end{abstract}

PACS: 73.20.-r Electron states at surfaces and interfaces;

73.20.Mf Collective excitations (including excitons, polarons, plasmons and other charge-density excitations).

Keywords: surface, liquid helium, magnetoplasmons.

\section{Introduction}

In bound two-dimensional electron systems (2DESs) placed in a strong perpendicular magnetic field, electrons in the vicinity of the system edges play an important role in influencing the system's transport properties. For example, edge electronic states significantly contribute to the magnetotransport of a degenerate 2DES in the quantum Hall regime [1,2] where the entire current is carried by edge electrons. An interesting application for spintronics devices proposes the use of edge electrons in graphene with zigzag edges, which support spin-dependent transport [3].

In this light, the important discovery of collective excitation modes was made in a classical (non-degenerate) 2DES formed on a liquid-helium surface $[4,5]$. The collec- tive excitation is called an edge magnetoplasmons (EMPs), in which an oscillation of charge fluctuation localizes in a very narrow strip near the edge of a 2DES and the charge oscillation propagates along the perimeter. EMPs appear as the lower frequency branch of 2D magnetoplasmons, and the frequency decreases as the external perpendicular magnetic field is increased. Subsequently, EMPs were observed in a wide variety of 2DESs including degenerate 2DESs in semiconductor heterostructures [6], 2DESs in nanostructures [7], and in 2D ion systems trapped under a liquid-helium surface [8]. Taking advantage of the EMP property that the charge oscillation localizes near the edge, researchers have attempt to employ EMPs as sensitive probes to study the structure of edge electronic states and electronic transport along edge channels $[9,10]$.

* Present address: Center of General Education, Tohoku Institute of Technology, Sendai 982-8577, Japan.

(C) Shuji Yamanaka, Toshikazu Arai, Anju Sawada, Akira Fukuda, and Hideki Yayama, 2013 
In EMPs, a deviation from the equilibrium electron distribution causes accumulation of charge in a narrow strip near the edge due to the Lorentz force acting on the electrons, and consequently, the EMP wave propagates along the perimeter [11,12]. The conventional EMP theory assumes that the EMP is a compression wave of the electron density near the edge, while the boundary position is fixed. The conventional EMP theory fairly accurately describes the basic features of EMPs. It is understood that the EMP spectrum is gapless, and thus, an EMP is a candidate for the lowest energy excitation from the ground state. One of the specific characteristics of an EMP is that its frequency is inversely proportional to the applied magnetic field. Therefore, at a sufficiently large value of magnetic field, EMP resonance can be observed at frequencies of the order $10 \mathrm{kHz}$ or less. Thus far, several types of EMP modes have been studied theoretically and experimentally [13]. Among the types of modes, there is one that propagates along the edges of an incompressible 2DES, and it is particularly observed in 2DESs in the quantum Hall states. In principle, an incompressible 2DES does not support the compression density wave. In such a case, the charge strip is induced by the displacement of the boundary from the equilibrium position while the uniform density distribution is maintained [14]. We call this mode as the boundary displacement wave (BDW). The 2DES formed on a liquid-helium surface is a classical non-degenerate 2DES, since the Fermi temperature $T_{F} \sim 10 \mathrm{mK}$ corresponding to the typical areal electron density $n \sim 10^{12} \mathrm{~m}^{-2}$ is considerably lower than the working temperature of standard experimental conditions ( $T>100 \mathrm{mK})$.

In general, a classical 2DES is not incompressible and the BDW is not responsible for the collective edge mode; however, it is predicted that the classical 2DES at low temperature can be nearly incompressible when the electron-electron Coulomb interaction energy dominates over the thermal kinetic energy of the electrons $[13,15]$. In the present paper, we report our observation of a novel BDW mode in a classical 2DES formed on a liquid-helium surface. We show that our novel BDW mode is an in-phase coupled oscillation of the conventional EMP and the BDW (BDW-EMP coupled mode, $\omega_{+}$), theoretically predicted by Monarkha [13,15]. Experimental observation of BDWEMP coupled mode has been reported as a small resonance peak whose frequency is slightly downshifted from the large resonance peak of a conventional EMP. The amount of the frequency shift in Ref. 16 was in reasonable agreement with theoretical calculation, and thus, the observed mode was identified as the out-of-phase coupled mode of the BDW-EMP $\left(\omega_{-}\right)$. In order to confirm that the BDWEMP coupled mode reflects the nearly incompressible feature of a classical 2DES, it is crucial to measure the damping rate of the resonance. The energy dissipation of the electron motion is determined by electron collision with the quanta of the capillary wave (ripplon) in the superfluid- helium surface. If the 2DES is nearly incompressible, electrons located in the deep interiors from the edge are in motion, and ripplon scattering of these electrons contributes to the dissipation. On the other hand, in the case of the compression wave of a conventional EMP, only the electrons within the narrow strip near the edge are responsible for the damping $[13,15]$. In the present work, we were able to transform the conventional EMP into the BDW-EMP coupled mode by lowering the strength of the electric field for lateral confinement. A pronounced increase was seen in the damping rate. We measured the enhanced damping rates of BDW-EMP coupled mode. Our results provide clear evidence of the nearly incompressible feature of the classical 2D Coulomb system.

This paper is organized as follows: In Sec. 2, a quick overview of EMPs is provided. In Sec. 3, we present our experimental techniques of EMP resonance and signal analysis. In Sec. 4, the experimental results concerning the enhanced damping rate are presented. In Sec. 5, we present our hypothesis that the oscillation mode with the enhanced damping rate is the BDW-EMP coupled $\omega_{+}$mode, and that the incompressible motion of the 2DES is responsible for the damping rate enhancement.

\section{Overview of edge excitation modes}

In this section, we provide a quick overview of EMPs. Owing to the characteristic properties listed below, EMPs have been extensively studied for various 2DESs both experimentally and theoretically. In the course of such studies several types of EMPs have been observed, such as the acoustic mode, boundary displacement wave (BDW), and the BDW-EMP coupled mode. These modes differ in their manner of charge oscillation; however, they have the following main characteristic properties in common: EMPs are gapless, i.e., $\omega_{E M P} \sim q \ln |q|$, where $\omega_{E M P}$ denotes the frequency, and $q$ denotes the wave number. Hence, EMPs is considered as the lowest energy excitation from the ground state of the 2DES. The EMP frequency is proportional to the 2DES density $n$ and inversely proportional to the applied normal magnetic field $B$, i.e., $\omega_{E M P} \propto n / B$. In contrast to the bulk 2D magnetoplasmons [5], the frequency of the EMPs decreases with increasing magnetic field. For sufficiently large value of $B$, $\omega_{E M P}$ is several orders of magnitude smaller than the cyclotron frequency $\omega_{c}=e B / m_{e}$, where $e$ and $m_{e}$ denote the charge and the mass of an electron, respectively. The EMP wave propagates along the perimeter of a bounded 2DES in a single direction determined by the sign of the Hall conductivity $\sigma_{x y}$. Accordingly, a resonance occurs when the perimeter length $P$ is an integer multiple of the wavelength, i.e., $q=2 \pi m / P(m=1,2, \ldots)$. These common properties can be understood within the conventional EMP model. 


\subsection{Conventional EMP}

Let us suppose that a charge fluctuation occurs in a 2DES. In applying a strong normal magnetic field, due to the Lorentz force, electrons move perpendicular to the electric field. In a bounded system, the charge fluctuation accumulates in a narrow strip near the edge and move only within the strip. A conventional EMP is a collective oscillation of electron density that propagates along the edges of a 2DES [4,5]. The conventional EMP theories of Volkov and Mikhailov [11,17] and Fetter [12,18,19] assume a fixed boundary position, and the charge fluctuation occurs as a density fluctuation. Therefore, the conventional EMP is a compression wave. Volkov and Mikhailov arrived at the expressions for the frequency $\omega_{E M P}$ and the damping rate $\gamma_{E M P}$ as below:

$$
\begin{gathered}
\omega_{E M P}=2 \pi f_{E M P}=-\frac{q \sigma_{x y}}{2 \pi \varepsilon}\left[\ln (|q| b)+C_{f}\right], \\
\gamma_{E M P} \sim-\frac{\sigma_{x x}}{4 \pi \varepsilon b} \frac{1}{\ln (|q| b)} .
\end{gathered}
$$

We denote the frequency of conventional EMP as $\omega_{E M P}$ hereafter. Here, $\varepsilon$ denotes the dielectric constant of the substrate; for the case of 2DES on liquid helium, $\varepsilon=1.057 \varepsilon_{0}$ is the dielectric constant of liquid helium $\left(\varepsilon_{0}\right.$ is the permittivity of vacuum). The parameter $b$ denotes the characteristic length scale of the charge strip where the EMPs localize. The term $\sigma_{x x}$ denotes the magnetoconductivity, and $\sigma_{x y}=n_{0} e / B$ is the Hall conductivity of the 2DES ( $n_{0}$ is the electron density at the center of the sample). The parameter $C_{f}$ denotes a geometrical constant.

\subsection{Acoustic modes}

In the theories of conventional EMPs, the equilibrium density profile near the edge is regarded as the stepfunction-shaped. In actual systems, the electron density falls off from $n_{0}$ to 0 at the edge with a characteristic length $w$. The step-function approximation holds as long as $b \gg w$. For the case of a 2DES on a liquid-helium surface, standard experiments involve a pair of parallel metalplate pressing electrodes above and below the surface. The pressing electrodes screen the electric field that is generated in the 2DES. The screening length $d$ is approximately the same as the distance between the 2DES and the pressing electrode, typically $\sim 1 \mathrm{~mm}$. Therefore, the oscillating EMP electric field does not penetrate deep within the 2DES over the distance $d$ so that the length $b$ may be of the same order as $d$. Likewise, the length scale $w$ is of the same order as $d$. Hence, for a 2DES on a liquid-helium surface, $b \sim w \sim d$. In such a situation, the step-function approximation becomes inaccurate and a more realistic equilibrium density profile is needed to account for the resulting profile. Nazin and Shikin [20] considered EMPs in a 2DES with a smooth equilibrium density profile near the edge, and they discovered a number of possible novel acoustic modes. In the acoustic mode, the charge density oscillates in the direction perpendicular to the edge, as well as in the direction along the edge. The spectrum of the acoustic mode changes according to the applied magnetic field. In weak magnetic fields, the frequency of the acoustic mode is calculated [20] to be proportional to $B$, and in strong magnetic fields, it is proportional to $1 / B$ as in the case of a conventional EMP [21]. The acoustic mode has been experimentally identified by Kirichek et al. [22]. Compared with the conventional EMP, a considerably smaller amplitude is expected to be observed in the acoustic mode, because of the screening effect by the alternating charge perpendicular to the edge [21].

\subsection{Boundary-displacement-wave (BDW) modes}

In the theories of the conventional EMP and the acoustic modes, it is assumed that the 2DES is a compressible Coulomb liquid. But in fact, EMP waves have been experimentally observed in incompressible 2DESs under the conditions of the quantum Hall effect [23]. By nature, the electron liquids in the quantum Hall regime are incompressible because of the presence of the excitation gap from the ground state. In the EMPs of incompressible 2DESs, charge fluctuation occurs as the boundary position displacement instead of the charge density fluctuation [14]. Hence, the edge wave that propagates in this manner is termed the boundary displacement wave. Although the origin of the charge fluctuation is different in the BDW from the conventional EMP, the restoring force for both modes is the Lorentz force acting on the electrons. Therefore, the BDW provides practically the same spectrum as the conventional EMP. Hence, from an experimental point of view, it is very difficult to distinguish a BDW from a conventional EMP by frequency measurement.

Intuitively, since classical electron liquids are in principle compressive, a BDW is not related in any way to the edge wave in a classical 2DES formed on a liquid-helium surface. However, it has been reported that the 2DES on liquid helium can be nearly incompressible at low temperatures, and the occurrence of a type of EMP mode along with boundary position motion is possible $[15,16,24]$. The theory predicts that when the Coulomb interaction energy between electrons dominates over the thermal kinetic energy, the strong repulsion force keeps the electrons apart and the 2DES can be nearly incompressible. In such a case, since the frequency of a BDW is close to that of a conventional EMP, these two modes can couple, thereby resulting in two novel modes: the in-phase and the out-of-phase BDW-EMP coupled modes, $\omega_{+}$and $\omega_{-}$modes, respectively. The spectra of the BDW-EMP coupled modes are calculated as

$$
\omega_{ \pm}=\gamma_{ \pm} \sigma_{x y} q
$$

where $\gamma_{ \pm}$denote dimensionless geometrical factors. The frequency $\omega_{+}\left(\omega_{-}\right)$appears at a slightly higher (lower) 
frequency value than that of the conventional EMP. In fact, the BDW-EMP coupled mode was experimentally identified by Kirichek et al. [16]. In the experiment, the authors of Ref. 16 observed small resonance peaks that were slightly downshifted to the lower frequency region apart from the main resonance peaks corresponding to conventional EMPs. The measured frequencies were in good agreement with the $\omega_{-}$values given by Eq. (3). The resonance amplitude of the out-of-phase BDW-EMP coupled oscillation should be small compared with that of the conventional EMP for the same reason that the amplitude of the acoustic mode is small [24]. The small observed amplitude of $\omega_{-}$also agrees with this qualitative picture.

A more significant difference between BDWs (including the BDW-EMP coupled modes) and conventional EMPs is expected to be observed in terms of the damping rate than in the frequency $[15,24]$. The number of electrons involved in the oscillation motion is large in BDWs because the incompressible motion of electrons penetrates deep towards the interior from the edge over the distance where the electric field fluctuation penetrates (screening length). The penetration depth of the incompressible electron motion is $q^{-1}$, in a similar manner to the fluid motion in gravity waves of water. On the other hand, in conventional EMPs, the electrons in motion are confined to the narrow charge strip in the vicinity of the edge. Therefore, the energy dissipation via the electron-ripplon collision and consequently the damping rate must be enhanced. The damping rate enhancement arising from the incompressible motion of a 2DES has not thus far been observed.

\subsection{Discussion on incompressible motion}

Among the two BDW-EMP coupled modes, the $\omega_{+}$ mode has not been experimentally identified. There is a suggestion that in the EMP resonance experiment of 2D ${ }^{4} \mathrm{He}^{+}$ion pool trapped below a liquid-helium surface $[25,26]$, the observed "extra satellites of unknown origin" may be the $\omega_{+}$mode [24]. In this respect, there is an argument about whether or not the BDW-EMP coupled mode with incompressible motion exists in classical 2D Coulomb systems. The author of Ref. 24 suggests that the higher shifted frequency of the satellites from the conventional EMP frequency may correspond to $\omega_{+}$. Against this suggestion, an argument was presented [26,27] based on an analysis comparing the amplitudes of electric field perturbation arising from boundary displacement and electron density compression. However, this analysis is questionable [24] in terms of the treatment of electron pressure.

The origin of the satellite mode is unclear, and the controversy over the incompressible motion is still unresolved. In order to clarify the nature of incompressible 2DES motion in classical 2D Coulomb systems, measurement of the enhanced damping rate in the BDW-EMP coupled mode is required.

\section{Experiment}

\subsection{Sample cell}

In order to study the nearly incompressible feature of a classical 2DES formed on liquid helium arising from strong electron-electron interaction, we designed our experiment in a manner that allows us to transform the conventional EMP into the BDW-EMP coupled mode and measure the difference in the damping rate.

Our sample cell is made of ordinary copper, and it is attached to the mixing chamber of a dilution refrigerator. We ensured that the cell was perfectly horizontally mounted. High purity (>99.99995\%) ${ }^{4} \mathrm{He}$ gas is condensed in the sample cell. The liquid surface level in the sample cell is carefully aligned with the center of a $6 \mathrm{~T}$ superconducting solenoid. The sample gas is filled from a room-temperature gas cylinder through a $0.7-\mathrm{mm}$-inner-diameter $\mathrm{Cu}-\mathrm{Ni}$ filling tube. During the filling process, the sample cell temperature is maintained below $1 \mathrm{~K}$, for which temperature the vapor pressure of ${ }^{4} \mathrm{He}$ is sufficiently low, in order to avoid shift in the liquid level by residual gas condensation on further cooling. Figure 1 shows the structure of the sample cell. The liquid-helium depth in the sample cell is precisely measured from the capacitance between the top and bottom electrodes. The top and bottom electrodes are circular in shape, $25 \mathrm{~mm}$ in diameter, and $3 \mathrm{~mm}$ apart. All data presented in this paper were measured under the condition that the depth $d$ from the bottom electrode was constant at $1.6 \mathrm{~mm}$.

The tungsten filament attached to the upper electrode is an electron source. The electrons emitted from the filament accumulate on the liquid-helium surface. The filament is made from a tiny light bulb from which the glass cover is removed. The typical operating condition to charge the surface is $0.6 \mathrm{~V} / 10 \mathrm{~mA}$ with a duration of $1 \mathrm{~s}$. To charge the surface, it is necessary to slow down the energetic thermoelectrons on the path from the filament to the sur-

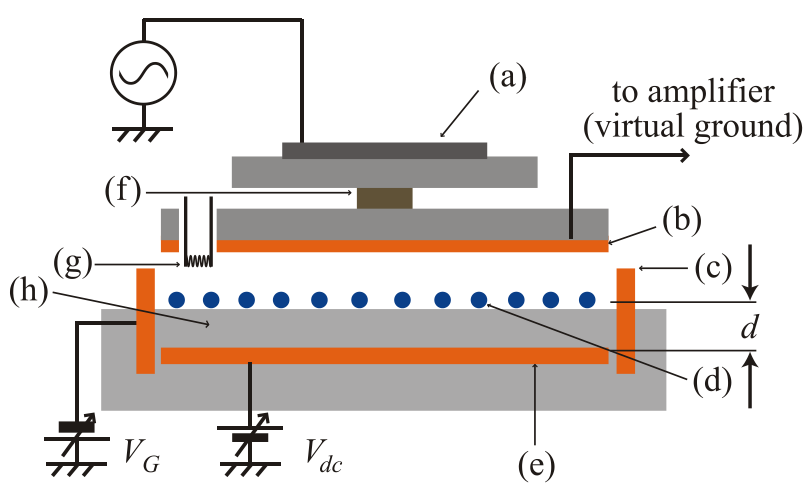

Fig. 1. (Color online) Electrode arrangement of the sample cell: (a) piezo actuator, (b) top electrode, (c) guard ring, (d) electrons, (e) bottom electrode, (f) mechanical connection supporting (a) and (b), (g) tungsten filament, (h) liquid ${ }^{4} \mathrm{He}$. 
face. Electrons possessing larger kinetic energy than the surface potential barrier $(\sim 1 \mathrm{eV})$ penetrate the liquid. Repeated collisions of electrons with cold helium vapor atoms during the flight can reduce the kinetic energy of electrons. To ensure repeated collision, we perform electron emission at a temperature close to $1.5 \mathrm{~K}$, at which temperature helium vapor is abundant.

The bottom electrode is biased by a positive dc source $V_{d c}=10-200 \mathrm{~V}$, while the top electrode is always grounded. The generated vertical electric field presses electrons onto the helium surface. The top electrode is mechanically connected to a piezo actuator. The application of an ac voltage to the piezo actuator causes vertical vibration of the top electrode. The estimated amplitude of vibration of $\sim 10^{-5} \mathrm{~m}$ is small compared with the distance between the top electrode and the surface $\left(\sim 10^{-3} \mathrm{~m}\right)$. Combined with the positively biased lower electrode, this assembly serves as a vibrating capacitor electrometer (VCE), which measures the total charge on the surface [28].

The electric field created by the guard ring electrode (26 mm inner diameter) surrounding the 2DES laterally confines the 2DES. By varying the applied potential $V_{G}$, which is normally negative, the strength of the lateral confinement electric field is controlled. A large negative value of $V_{G}$ corresponds to strong confinement. The value of $V_{G}$ changes the electron density distribution near the edge. The curves shown in Fig. 2 are the numerically calculated electron density distributions $n(r)$, where $r$ denotes the radial coordinate, for our sample cell geometry and applied electrode potentials. The electron density distribution is uniform in the interior and falls off near the edge. We define $R_{e}$ as the 2DES radius at which $n(r)$ drops to zero. The density distribution curves are calculated under the boundary conditions of realistic experimental parameters: $V_{G}=-10 \mathrm{~V}$ (small radius) and $0 \mathrm{~V}$ (large radius), respectively, for a given $V_{d c}=74 \mathrm{~V}$, and the total number of

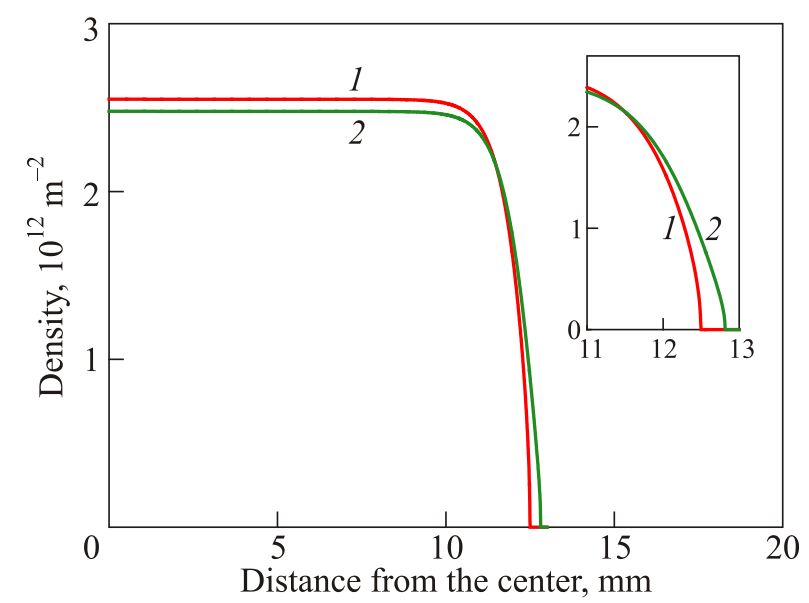

Fig. 2. (Color online) Calculated electron density profiles in the (1) — strong (red) and (2) — weak (green) confinement regimes for the immersed-guard-ring case. Inset: enlarged view at the edge. electrons $N_{e}=1.16 \cdot 10^{9}$, and these are conditions corresponding to the resonance spectra shown in Fig. 4. As the lateral confinement field is decreased, the 2DES expands, and, therefore, $R_{e}$ increases and the central density $n_{0}$ becomes small. For a given surface electron density $n$, Gauss's law determines the potential $V_{e}$ of the 2DES. In this work, all measurements were carried out under unsaturated electron densities, i.e., $V_{e}>0 \mathrm{~V}$. The electrons are confined without loss as long as $V_{e}>V_{G}$.

\subsection{EMP resonance technique}

We employed the standard frequency sweep continuous-wave EMP resonance technique in our experiment [29]. The EMP signals are measured via the capacitive coupling between the 2DES and the bottom electrode [30]. The bottom electrode is divided into five segments by using $0.1 \mathrm{~mm}$ spacings, as illustrated in Fig. 3. The electrode array was photo-etched on a glass-epoxy printed circuit board. The area of the central disk $\mathrm{E}$ is half of that of the entire disk, as are the areas of the segments A-D. As shown in Fig. 3, all the segments of the bottom electrode are equally biased by $V_{d c}$ through identical resistors of $R=10 \mathrm{M} \Omega$. The input ac voltage to the segment A drives charge oscillations near the edge. The charge oscillations propagate as EMP waves along the 2DES perimeter and are detected as an ac current in segment $C$. The inserted blocking capacitor $C_{B}$ isolates the ac signals from $V_{d c}$. The time constant $R C_{B}=1 \mathrm{~s}$ is sufficiently large compared with the period of ac signals $\left(<10^{-4} \mathrm{~s}\right)$, thereby making the signal current leakage in the circuit of $V_{d c}$ negligible. We measured the signal current amplitude as a function of the excitation frequency $f$. Resonance occurs when the 2DES perimeter is an integer multiple of the EMP wavelength. Too high a driving voltage sets off a nonlinear transport of 2DES, as reported by Monarkha et al. [31]. We varied the driving voltage and determined a value such that the EMP signals behaved well in the linear transport regime.

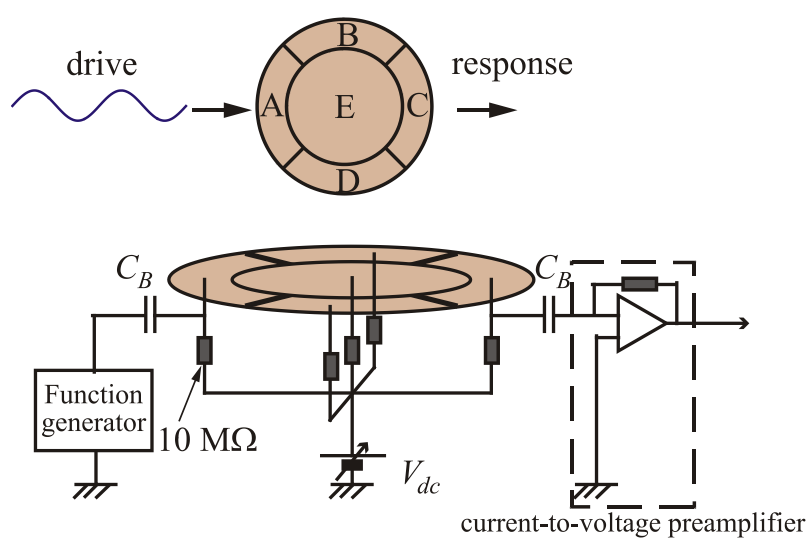

Fig. 3. (Color online) Schematic explanation of the edge magnetoplasmon (EMP) resonance experiment electronics and the divided bottom electrode. 
We systematically investigated the influence of the $V_{G}$ variation on the EMP spectra. As mentioned previously, $V_{G}$ determines the strength of the lateral confinement electric field. When comparing a conventional EMP and a BDW (or the BDW-EMP coupled mode), it is easy to assume that the BDW is favored in weak lateral confinement conditions because the energy cost for the boundary displacement against the confining electric field can be low. During a series of measurements, we carefully maintained constant the values of $T, B$, and the total number of electrons $N_{e}$. Since maintaining $N_{e}$ constant is of prime importance in this work, we carefully checked the reproducibility of the spectra.

\subsection{Signal analysis}

Figure 4 shows our resonance spectra for $V_{G}=-10$ and $0 \mathrm{~V}$, measured at $T=0.58 \mathrm{~K}$ and $B=3.2 \mathrm{~T}$. The resonance condition for the $m$ th harmonic is given as $2 \pi R_{e}=m \lambda$, where $\lambda$ denotes the wavelength. Clear resonances of the first few harmonics are visible for both values of $V_{G}$.

We assume simple damped harmonic oscillations for the EMP resonance and fit the first two resonance peaks $(m=1,2)$ to the following function $F(\omega)$ :

$$
F(\omega)=\frac{A_{1}(\omega)+A_{2}(\omega)}{\sqrt{R^{2}+(\omega L-1 / \omega C)^{2}}}
$$

with $A_{m}(\omega)=a_{m} / \sqrt{\left(\omega^{2}-\omega_{m}^{2}\right)^{2}+\gamma_{m}^{2} \omega^{2}}, \omega=2 \pi f$ denotes the driving angular frequency, $\omega_{m}$ denotes the resonant angular frequency, $\gamma_{m}$ denotes the damping rate, $a_{m}$ denotes the amplitude parameter, and $R, L$, and $C$ represent the

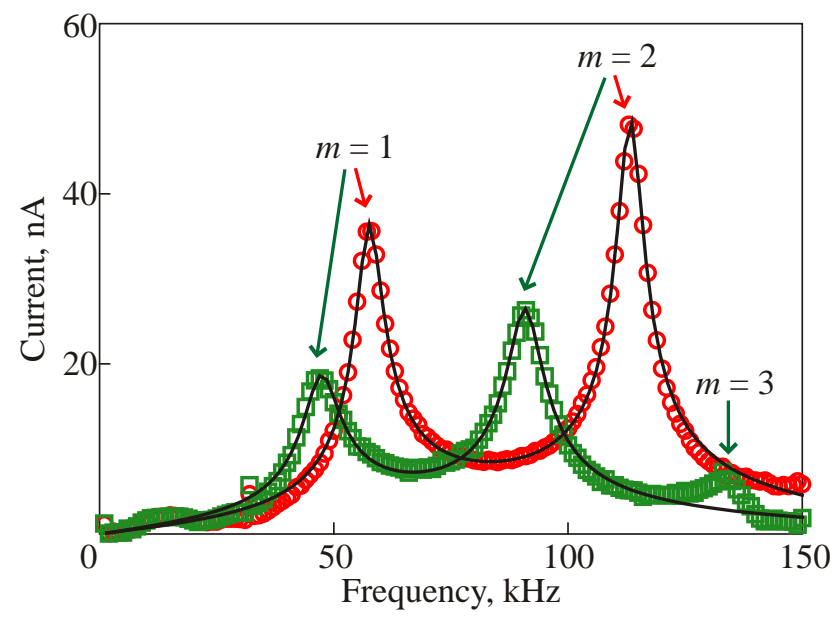

Fig. 4. (Color online) EMP spectra for strong $\left(V_{G}=-10 \mathrm{~V}\right.$, red circles) and weak $\left(V_{G}=0 \mathrm{~V}\right.$, green squares) lateral confinements. The black solid lines indicate the fitting results of $F(\omega)$. The conditions for acquiring the spectra are following: $T=0.55 \mathrm{~K}$, $B=3.2 \mathrm{~T}, N_{e}=1.16 \cdot 10^{9}$, and the distance from the bottom electrode and the liquid surface $d=1.6 \mathrm{~mm}$. The guard ring is immersed in the liquid helium. Electrons are emitted at $V_{d c}=70 \mathrm{~V}$ and the measurements are performed at $V_{d c}=74 \mathrm{~V}$. external circuit resistance, inductance, and capacitance, respectively. Since the quantity being measured is the electric current, it is necessary to divide the voltage $A_{1}(\omega)+A_{2}(\omega)$ by the external circuit impedance. We adjusted the values of $\omega_{m}, \gamma_{m}$, and $a_{m}$ as fitting parameters, while $R, L$, and $C$ were fixed. As shown by the solid curves in Fig. 4, the fitting results reproduce the spectra well.

A frequency shift to the lower side is observed in the weak confinement spectrum (green squares in Fig. 4). This shift can be intuitively understood as the consequence of the enlarged 2DES edge perimeter, corresponding to an increase in the wavelength in the weak lateral confinement electric field. The resonance peak amplitudes of the weak confinement spectrum in Fig. 4 appear to be suppressed compared with those of the strong confinement spectrum. However, this suppression is due to the external circuit impedance factor in Eq. (4). The impedance increases with increasing $\omega$ within our frequency range of interest. It should be emphasized that there is no significant difference in the intrinsic amplitude of the numerator of Eq. (4), regardless of variation in the values of $V_{G}$.

\section{Results and analysis}

Figure 5 shows the $V_{G}$ dependence of (a) the resonance frequency, (b) the damping rate $\gamma$, and (c) the $Q$-value

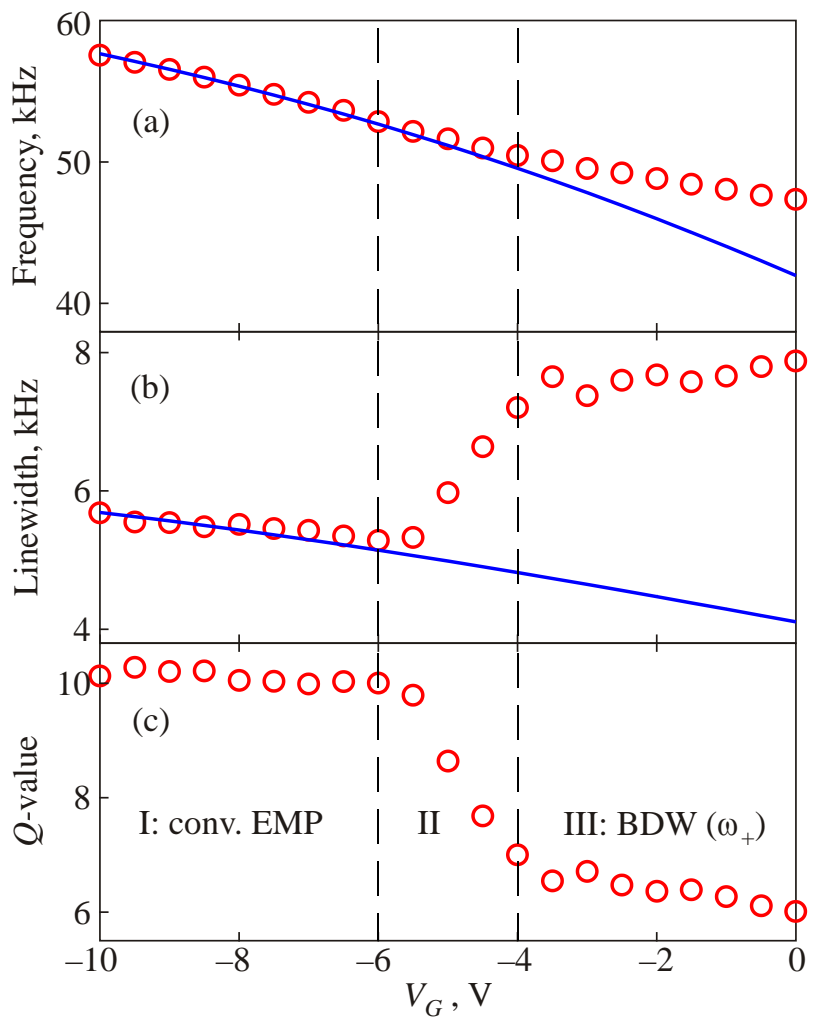

Fig. 5. (Color online) Relation between $V_{G}$ and frequency (a), linewidth (b), and $Q$-value (c). The red open circles indicate the experimental results $\left(T=0.55 \mathrm{~K}, B=3.2 \mathrm{~T}, N_{e}=1.16 \cdot 10^{9}\right.$, and $\left.V_{d c}=74.0 \mathrm{~V}\right)$. The blue solid lines indicate the numerical conventional EMP frequency and linewidth from the Volkov and Mikhailov theory [17]. 
$\left(f_{1} / \gamma_{1}\right)$ for the first resonance peaks. Since the behavior of the second peak is essentially the same as that of the first peak, only the first-peak results are shown.

The most striking behavior is observed in the damping rate shown in Fig. 5(b). In the course of decreasing $\left|V_{G}\right|$, the damping rate reaches a minimum value near $V_{G}=-6 \mathrm{~V}$, and it increases in the intermediate region II while finally reaching the limiting value at $V_{G}=-4 \mathrm{~V}$ (region III). In a similar manner, the $Q$-value changes from about 10 in region I to 6 in region III. The behavior of the damping rate as well as the $Q$-value apparently indicates that the damping mechanisms for region I and III are different. The resonance frequency monotonically reduces with decreasing $\left|V_{G}\right|$, but a kink is observed at around $V_{G}=-4 \mathrm{~V}$.

We define the region boundaries as the point at which the damping rate is minimum (I-II boundary) and the point at which the frequency curve shows the kink (II-III boundary). We hypothesize that an oscillation mode transformation occurs from region I to III across the intermediate region II. Decrease in the lateral confinement electric field results in an expansion of the 2DES and thus, the 2DES edge approaches the guard ring electrode. If the top of the guard ring electrode is positioned upright, as shown in Fig. 1, above the surface, the influence of the surface deformation of the meniscus will also need to be considered. In order to examine the influence of the surface deformation, we compared the EMP spectra using two types of guard ring: one was upright (half-immersed in the liquid) and the other was completely immersed in the liquid helium, thereby ensuring the liquid surface was flat. The oscillation mode transformation was observed in both configurations, and therefore, the meniscus is not a factor in our experiment.

\section{Discussion}

Let us consider the oscillation mode in region I. Since the electrons are strongly confined in the lateral direction, it is reasonable to assume the occurrence of a conventional EMP. The curves in Figs. 5(a) and (b) indicate the calculated values of the conventional EMP. We used the Eqs. (1) and (2) for our calculations. We see that the Volkov and Mikhailov $[11,17]$ equations well reproduce the experimental results for region I. In the calculation, we arbitrarily assumed $b$ as the distance from $R_{e}$ to the radius where $n(r)$ rises up to $50 \%$ of the central density $n_{0}$. This assumption is justified for the following reason: The top and bottom pressing electrodes screen the oscillating electric field of the charge strip with a screening length $d$, which is the distance between the 2DES and the bottom electrode, and the width of the charge strip $b$ is estimated to be of the same order as $d$. The characteristic length scale of the $n(r)$ change near the edge is also of the order of $d$. The $C_{f}$ and $\sigma_{x x}$ values were adjusted to fit the data. For the purpose of confirmation, the obtained $\sigma_{x x}$ values were compared with

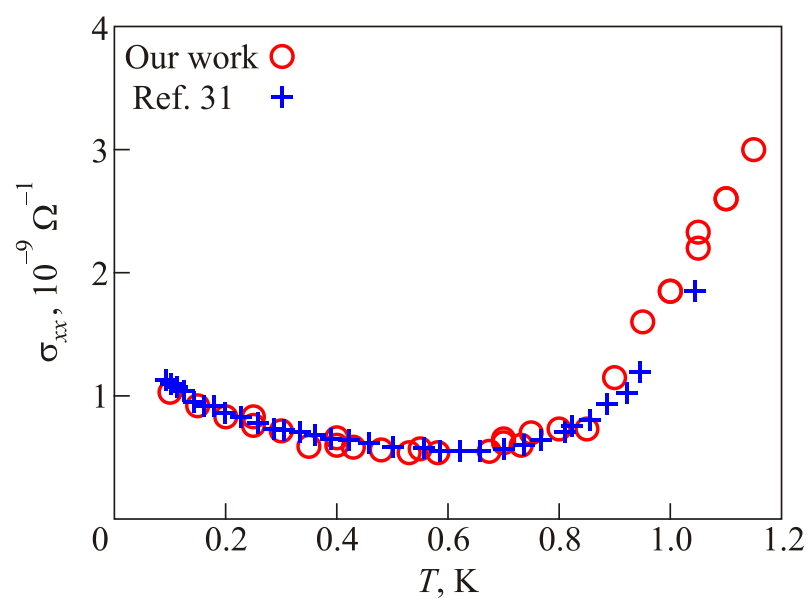

Fig. 6. (Color online) Comparison of our results (red circles) with the results in Ref. 31 (blue pluses) as regards the relation between temperature and $\sigma_{x x}$. In order to compare the $\sigma_{x x}$ values obtained for different electron density and magnetic field, we assumed Drude model of magnetoconductivity and the $\sigma_{x x}$ data of Ref. 31 inelastic are multiplied by a factor of 2.4.

the result of an earlier experiment [31] measured at $n_{0}=3.15 \cdot 10^{11} \mathrm{~m}^{-2}$ and $B=1.84 \mathrm{~T}$. We measured the $\sigma_{X x}$ values of Fig. 6 under conditions of $n_{0}=2.5 \cdot 10^{12} \mathrm{~m}^{2}$ and $B=3.19 \mathrm{~T}$. In order to compare the $\sigma_{x x}$ data obtained for different values of $n_{0}$ and $B$, we assume the result of Drude model of magnetoconductivity in the limit of strong magnetic field, $\sigma_{x x} \propto n_{0} / B^{2}$. According to the Drude model, the $\sigma_{x x}$ values of Ref. 31 are required to be scaled by multiplying a factor of 2.4. As shown in Fig. 6, the behavior of our obtained $\sigma_{x x}$ curve is in good agreement with the result of an earlier experiment [31]. Therefore, the oscillation mode of region I can be regarded as corresponding to the conventional EMP. We note that the $\sigma_{x x}$ of 2DES on liquid helium at low temperatures is known to be not fully described within the simple Drude model and it requires more sophisticated theory [31]. Taking that into account, the coincidence of the scaling factor of 2.4 could be accidental.

The oscillation mode of the weak-lateral-confinement region III is expected to be the BDW. The enhanced damping rate agrees with the qualitative prediction in the study by Monarkha [13], in which it is pointed out that the incompressible electron motion of the BDW penetrates deep within the interior of the 2DES beyond the screening length where electric field perturbation is absent; this penetration results in a strong damping, while the electron motion in the conventional EMP is confined within the screening length. Since the 2DES on helium in practice remains compressible, the BDW couples with the conventional EMP in strong magnetic fields [15,16]. The wave that we considered as the BDW would consequently correspond to the BDW-EMP coupled mode. 

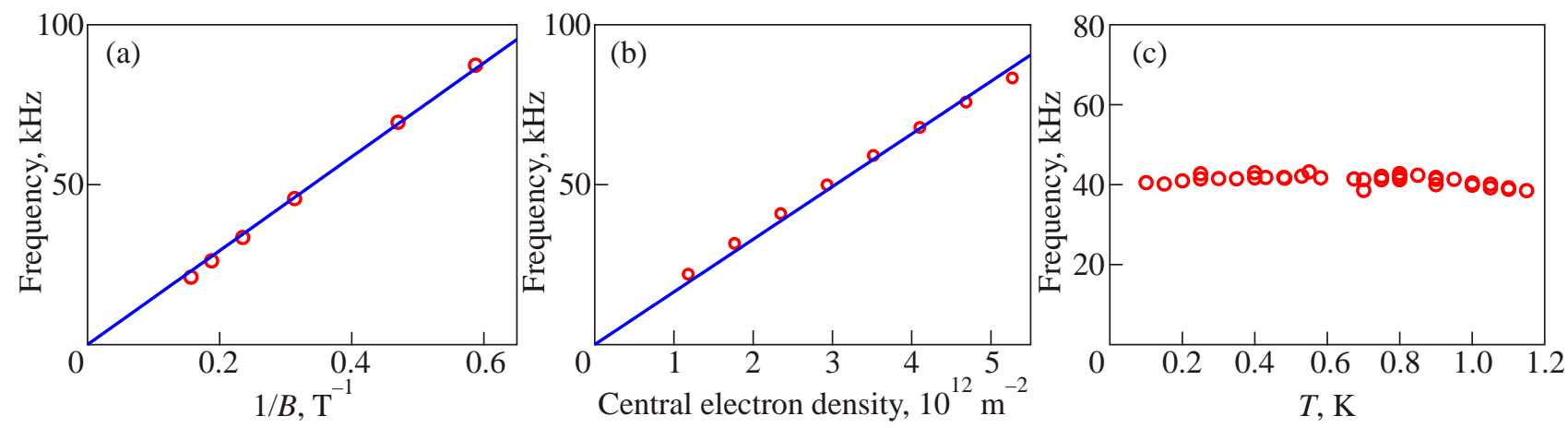

Fig. 7. (Color online) Measured first-peak frequencies vs $1 / B$, the central electron density, and temperature in the weak confinement regime $\left(V_{G}=0 \mathrm{~V}\right)$. The blue solid lines indicate the values obtained using the least mean square method. The guard ring is immersed $(d=1.6 \mathrm{~mm})$ and $V_{d c}=74 \mathrm{~V}$. The other conditions are (a): $T=0.55 \mathrm{~K}$ and $N_{e}=1.16 \cdot 10^{9}$, (b): $B=3.2 \mathrm{~T}$ and $T=0.55 \mathrm{~K}$, (c): $B=3.2$ T and $N_{e}=1.16 \cdot 10^{9}$.

The BDW-EMP coupled mode was first observed by Kirichek et al. [16]. Upon comparison of our results with the resonance signals obtained in Ref. 16, there are both similarities and differences. The appearance of the BDWEMP coupled mode in Ref. 16 is characterized by separate small resonance peaks in the lower frequency domain away from the conventional EMP main peaks. The frequency is proportional to $1 / B$, in the same manner as that of the conventional EMP. The coupled mode was observed only at $T<0.9 \mathrm{~K}$, where the damping is sufficiently small; however, the damping rate was not measured quantitatively. The above-mentioned observations were successfully explained by the out-of-phase BDW-EMP coupling (the $\omega_{-}$mode) $[15,16]$. Similarly, our resonance frequency in region III varies as $1 / B$ (Fig. 7(a)) and the oscillation mode transformation is clear at $T<0.9 \mathrm{~K}$ (Fig. 8). At higher temperatures, it becomes difficult to recognize the change in the damping rate.

Our resonances in region III shows some differences. We observed the BDW as a single resonance peak, in contrast to the separate peaks observed in Ref. 16. This indi-

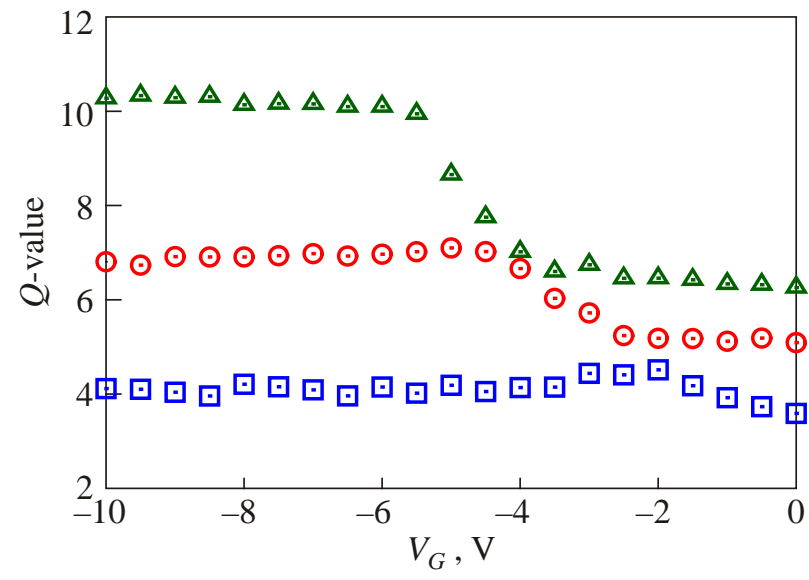

Fig. 8. (Color online) $V_{G}$ vs $Q$-value at various temperature values ( $B=3.2 \mathrm{~T}$ and $N_{e}=1.16 \cdot 10^{9}$ ). The temperature values are 0.28 (red circles), 0.58 (green triangles) and $0.88 \mathrm{~K}$ (blue squares). The guard ring is immersed $(d=1.6 \mathrm{~mm})$. cates that the oscillation mode in our case transforms from the conventional EMP of region I into the BDW-EMP coupled mode of region III depending on the lateral confining electric field. When compared with the conventional EMP, in our case, the frequency is higher and the amplitude is of the same order. The comparison of our results and the reported behavior of $\omega_{-}$[16] is summarized in Table 1.

Table 1. Comparison of other EMP modes observed in our study and Ref. 16

\begin{tabular}{l|c|c}
\hline \hline \multicolumn{1}{c|}{ Parameter } & This work & Ref. 16 \\
\hline \hline Appearance & single peak & separate peak \\
Amplitude & identical & considerably smaller \\
Temperature & $T \lesssim 0.9 \mathrm{~K}$ & $T \lesssim 0.9 \mathrm{~K}$ \\
Frequency & $\propto n_{0} / B$ & $\propto n_{0} / B$ \\
Damping & strong & no data \\
Identification & $\omega_{+}$ & $\omega_{-}$ \\
\hline \hline
\end{tabular}

Upon evaluating these observations together, along with the enhanced damping rate, we conclude that the oscillation mode in region III is consistent with the in-phase BDW-EMP coupled mode (the $\omega_{+}$mode) [15]. Our identification of the $\omega_{+}$mode is further confirmed from the dependence of the frequency on the magnetic field (Fig. 7(a)), central density (Fig. 7(b)), and temperature (Fig. 7(c)). All our observations from Fig. 7 are consistent with the behavior expressed by Eq. (3); $\omega_{+}$is proportional to $n_{0} / B$ and independent of temperature. The observation of the $\omega_{+}$mode has not yet been confirmed. There has been a speculation that the "satellite mode" in Ref. 15 observed in a 2D ion pool trapped below a liquid-helium surface may be the $\omega_{+}$mode $[13,15]$; however, the speculation is still controversial [26,27] and the origin of the satellite mode is still unclear.

Our observation of the enhanced damping rate supports the hypothesis by Monarkha $[13,15]$. The electron correlation of classical electron liquids becomes strong for high densities, in contrast to quantum electron liquids that tend 
to behave as an ideal Fermi gas in the high density limit. To examine the relationship between the incompressibility and electron correlation, we measured the region boundaries of the oscillation modes at various electron densities. From the $V_{G}$ values for the measured region boundaries, we calculated the central electron density $n_{0}$ and the average lateral confining electric field $E_{G}$ at the outer proximity of the 2DES for the equilibrium conditions.

The field $E_{G}$ was calculated as follows [32]. For a given $V_{G}$ value, we solved the electrostatic equation for the potential $\phi(r)$ of the helium surface level under appropriate boundary conditions. For a small distance $\Delta r$ outside the edge, we define $E_{G}$ as the average gradient of $\phi(r)$ :

$$
E_{G}=\left|-\frac{\phi\left(R_{e}+\Delta r\right)-\phi\left(R_{e}\right)}{\Delta r}\right| .
$$

We assumed $\Delta r=20 \mu \mathrm{m}$ for the sake of numerical precision. At equilibrium, the expanding force of Coulomb repulsion within the 2DES and the confining force caused by the guard electrode field acting on the electrons at the edge are balanced so that $E_{G}=0$ for $\Delta r \rightarrow 0$ [13]. The $E_{G}$ value obtained for a finite $\Delta r$ value provides a measure of the strength of lateral confinement.

Figure 9 shows the $E_{G}$ values at the mode transformations as a function of $n_{0}$. The filled circles and triangles represent the experimentally obtained boundaries of regions I-II and II-III, respectively, and the lines show the linear fit of these values. These values were measured using the half-immersed guard ring configuration. The results for the I-II boundary region for the completely immersed guard ring configuration are also plotted (open circles). It can be observed that the lines dividing the oscillationmode regions are independent of the sample cell geometry, thereby indicating that the curves in Fig. 9 indicate the

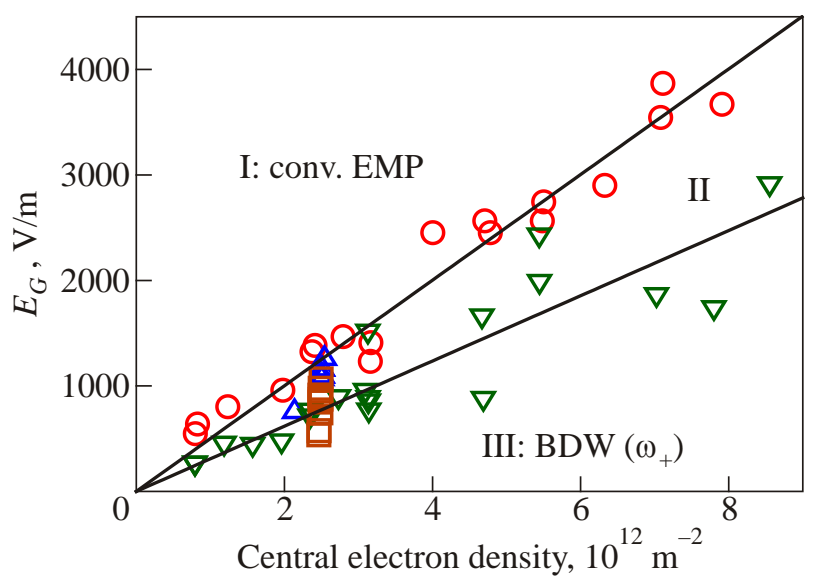

Fig. 9. (Color online) Confining lateral electric field $E_{G}$ of the edge mode transition points vs. the central electron density. The red circles and the inverted green triangles indicate the data for the immersed guard ring case. The blue triangles and orange squares indicate the data for the half-immersed-guard-ring case. The straight line indicates the result of the least mean square fit. universality of the oscillation mode for given values of $n_{0}$ and $E_{G}$. The appearance of the BDW in the strong confinement regime at high densities suggests that the strong electron correlation gives rise to the incompressibility of the classical 2DES.

Our results can be explained by the following simple analysis. The compressibility $\kappa$ is related to the density $n$ and the chemical potential $\mu(n)$ by the relation

$$
\kappa^{-1}=n^{2} \frac{d \mu}{d n} .
$$

Using Seitz's theorem, $\mu(n)=\varepsilon_{G}+n\left(d \varepsilon_{G} / d n\right)$ and the ground-state energy [33] $\varepsilon_{G} \sim-n^{1 / 2}$, we have $\kappa \sim-n^{-3 / 2}$. Therefore, the compressibility would be small for large values of $n$.

\section{Conclusion}

In summary, we obtained clear evidence of an oscillation mode transformation from the conventional EMP into the BDW-EMP coupled mode in a classical 2DES formed on the surface of liquid helium. The latter mode appears when the strength of the lateral confinement electric field is weak. The behavior the BDW-EMP coupled mode is consistent with the theoretical prediction of the in-phase coupled mode $\left(\omega_{+}\right.$mode) [15]. The coupling of the EMP with the BDW enhances the damping of the oscillation, thereby indicating the incompressibility arising from the strong correlation in the classical Coulomb liquid.

\section{Acknowledgments}

We would like to thank K. Kono for his attention to our work and for helpful discussions. We would like to express our gratitude to R. Nishinakagawa and S. Isoyama for their assistance with our experiments. This work was supported by a Grant-in-Aid for Challenging Exploratory Research from the Ministry of Education, Culture, Sports, Science and Technology (MEXT), Japan (Research No. 16654058) and by a Grant-in-Aid for Scientific Research (B) from Japan Society for the Promotion of Science (JSPS) (Nos. 16340108, 19540337, and 21540319).

1. B.I. Halperin, Phys. Rev. B 25, 2185 (1982).

2. M. Büttiker, Phys. Rev. B 38, 9375 (1988).

3. O.V. Yazyev and M.I. Katsnelson, Phys. Rev. Lett. 100, 047209 (2008).

4. D.C. Glattli, E.Y. Andrei, G. Deville, J. Poitrenaud, and F.I. B. Williams, Phys. Rev. Lett. 54, 1710 (1985).

5. D.B. Mast, A.J. Dahm, and A.L. Fetter, Phys. Rev. Lett. 54, 1706 (1985).

6. I.M. Grodnensky, D. Heitmann, K. von Klitzing, and A.Y. Kamaev, Phys. Rev. B 44, 1946 (1991).

7. T. Demel, D. Heitmann, P. Grambow, and K. Ploog, Phys. Rev. Lett. 64, 788 (1990). 
8. N.J. Appleyard, G.F. Cox, L. Skrbek, P.K.H. Sommerfeld, and W.F. Vinen, Phys. Rev. B 51, 5892 (1995).

9. V.I. Talyanskii, A.V. Polisski, D.D. Arnone, M. Pepper, C.G. Smith, D.A. Ritchie, J.E. Frost, and G.A.C. Jones, Phys. Rev. B 46, 12427 (1992).

10. M.N. Khannanov, A.A. Fortunatov, and I.V. Kukushkin, JETP Lett. 93, 657 (2011).

11. V.A. Volkov and S.A. Mikhailov, Sov. Phys. JETP 67, 1639 (1988).

12. A.L. Fetter, Phys. Rev. B 33, 5221 (1986).

13. Edge Excitations of Low-Dimensional Charge Systems (Horizons in World Physics), O. Kirichek (ed.), Nova Science Publishers, Inc. (2001).

14. S. Giovanazzi, L. Pitaevskii, and S. Stringari, Phys. Rev. Lett. 72, 3230 (1994).

15. Yu.P. Monarkha, Fiz. Nizk. Temp. 21, 589 (1995) [Low Temp. Phys. 21, 458 (1995)].

16. O.I. Kirichek, P.K.H. Sommerfeld, Yu.P. Monarkha, P.J.M. Peters, Y.Z. Kovdrya, P.P. Steijaert, R.W. van der Heijden, and A.T.A.M. de Waele, Phys. Rev. Lett. 74, 1190 (1995).

17. V.A. Volkov and S.A. Mikhailov, Electrodynamics of TwoDimensional Electron Systems in High Magnetic Fields, Chap. 15, in: Landau Level Spectroscopy, G. Landwehr and E.I. Rashba (eds.), Elsevier Science Publishers B. V. (1991).

18. A.L. Fetter, Phys. Rev. B 32, 7676 (1985).

19. A.L. Fetter, Phys. Rev. B 33, 3717 (1986).
20. S.S. Nazin and V.B. Shikin, Sov. Phys. JETP 67, 288 (1988).

21. I.L. Aleiner and L.I. Glazman, Phys. Rev. Lett. 72, 2935 (1994).

22. O.I. Kirichek, I.B. Berkutov, Y.Z. Kovdrya, and V.N. Grigor'ev, J. Low Temp. Phys. 109, 397 (1997).

23. V.A. Volkov, D.V. Galchenkov, L.A. Galchenkov, I.M. Grodnenskii, O.R. Matov, and S.A. Mikhailov, JETP Lett. 44, 655 (1986).

24. Yu. Monarkha, in Ref. 13.

25. P.L. Elliott, C.I. Pakes, L. Skrbek, and W.F. Vinen, Phys. Rev. Lett. 75, 3713 (1995).

26. P.L. Elliott, S.S. Nazin, C.I. Pakes, L. Skrbek, W.F. Vinen, and G.F. Cox, Phys. Rev. B 56, 3447 (1997).

27. V.B. Shikin, S.S. Nazin, L. Skrbek, and W.F. Vinen, J. Low Temp. Phys. 110, 237 (1998).

28. T. Arai, A. Würl, P. Leiderer, T. Shiino, and K. Kono, Physica B 284, 164 (2000).

29. T. Arai, S. Yamanaka, H. Yayama, A. Fukuda, and A. Sawada, J. Phys.: Conf. Ser. 150, 032129 (2009).

30. W.T. Sommer and D.J. Tanner, Phys. Rev. Lett. 27, 1345 (1971).

31. Yu.P. Monarkha, S. Ito, K. Shirahama, and K. Kono, Phys. Rev. Lett. 78, 2445 (1997).

32. R. Mehrotra, J. Low Temp. Phys. 67, 123 (1987).

33. Y. Monarkha and K. Kono, Two-Dimensional Coulomb Liquids and Solids, Springer-Verlag (2004). 